\title{
APORTACIONES DESDE LA PSICOLOGÍA AL TRATAMIENTO DE LAS PERSONAS CON INFECCIÓN POR VIH/SIDA
}

\author{
RAFAEL BALLESTER
}

\author{
Universidad Jaime I de Castellón
}

\begin{abstract}
En este artículo se pretende exponer la situación actual por lo que respecta a las aportaciones desde la Psicología al tratamiento de las personas con infección por VIH/SIDA. Tras exponer los últimos datos epidemiológicos que hablan de una enfermedad que sigue progresando a un ritmo alarmante, así como los importantes avances y limitaciones de las intervenciones preventivas y del tratamiento médico hasta el momento, en este trabajo se comentan los problemas emocionales que suelen afectar a las personas con VIH y la repercusión que los factores psicológicos tienen en la progresión de la enfermedad. Se hace una mención especial a una de las cuestiones más críticas en el cuidado de los pacientes, la adhesión al tratamiento, y se revisan los principales programas de intervención psicológica que han sido desarrollados para abordar este problema, así como para mejorar la calidad de vida de los enfermos. Finalmente, se apuntan los retos actuales en la investigación psicológica sobre el SIDA, incluyendo una mayor comprensión en los factores implicados en las conductas de riesgo, la evaluación de la eficacia de las acciones preventivas, la intervención psicológica en los niños y adolescentes afectados por el VIH, el análisis de la eficacia de los tratamientos psicológicos a los afectados y la atención a los familiares y personal sanitario que trata con estos enfermos.
\end{abstract}

Palabras clave: VIH/SIDA, psicología, tratamiento.

\section{Contributions from Psychology to treatment of people living with HIV/AIDS}

The aim of this paper is to explain the current situation related to contributions from Psychology to the treatment of people living with HIV/AIDS. After presenting the last epidemiological data showing an infection with an alarming progress and the important advances and limitations in the field of prevention and medical treatment up to now, we discuss the emotional problems that affect people living with HIV and the impact that psychological factors have on infection. A special mention is made to one major critical question in the care of these patients, the adherence to treatment, and the main psychological intervention programs to approach this problem and to improve quality of life of these patients. Finally, present challenges in psychological research on AIDS are emphasized including a better understanding of factors involved in risk behaviors, the evaluation of effectiveness of prevention programs, psychological intervention of children and adolescents infected with HIV, efficacy of psychological treatments for the patients and attention to their relatives and the health providers.

Key words: HIV/AIDS, psychology, treatment.

\section{SITUACIÓN ACTUAL DE LA INFECCIÓN POR VIH EN LO QUE RESPECTA A SU EPIDEMIOLOGIA, PREVENCIÓN Y TRATAMIENTO}

\section{Desde que el Virus de Inmunodeficiencia Humana fue aislado a principios de la}

Correspondencia: Rafael Ballester, Departamento de Psicología Básica, Clínica y Psicobiología, Universidad Jaime I de Castellón, Avda. Sos Baynat s/n, 12071 Castellón. Correo-e: rballest@psb.uji.es década de los 80 han sucedido muchos hechos que han cambiado notablemente la situación del mundo en relación con la epidemia del SIDA. En primer lugar, por lo que respecta a la progresión de la enfermedad, en apenas veinte años se ha pasado de los 41 casos detectados entre 1981 y 1982 a los 40 millones de casos estimados actualmente, lo que le confiere a esta enfermedad el carácter de pandemia que afecta a personas de absoluta- 
mente todo el mundo. En nuestro país, los últimos datos epidemiológicos indican que el número de infectados se sitúa en los 150.000, de los que más de 66.000 tienen SIDA. En segundo lugar, podemos decir que la enfermedad no sólo se ha extendido en cuanto al número de personas a las que afecta y también desde el punto de vista geográfico. También lo ha hecho en cuanto al tipo de personas que la padecen. Si los primeros casos correspondieron básicamente a hombres que habían mantenido relaciones homosexuales y a drogodependientes, en la actualidad la epidemia se ha universalizado, afecta a cualquier persona y precisamente es entre los heterosexuales no drogodependientes donde más se está extendiendo en estos momentos.

La rápida progresión de la enfermedad alertó a las autoridades sanitarias desde un principio y motivó que se invirtieran grandes esfuerzos y dinero en intentar prevenir nuevas infecciones, en comprender los mecanismos biológicos que hacían de la infección por VIH una enfermedad tan devastadora para el organismo humano y en desarrollar tratamientos farmacológicos que pudieran detenerla. Por lo que respecta a la prevención se observa una evolución que podríamos denominar "positiva" pero con muchos matices. En primer lugar, como decíamos, se ha hecho un enorme esfuerzo para informar a la población acerca de las vías de transmisión de la enfermedad, que como se sabe, son los fluidos corporales, especialmente la sangre, el semen y los fluidos vaginales. El hecho de que el VIH sea un virus cuyos mecanismos de propagación son fácilmente evitables a través del cambio de hábitos nos ha llevado demasiado ilusamente a pensar que su control era sencillo y que para ello bastaba con informar a la población. Sin embargo, la experiencia nos ha demostrado que pocas cosas son más difíciles que modificar la conducta humana. Tal como afirma ONU-
SIDA en su último comunicado de prensa con motivo del Día del SIDA (1 de Diciembre de 2003), "la epidemia mundial de SIDA no muestra signos de ceder, el número de infecciones por el VIH y de defunciones por SIDA es más alto que nunca». El número de nuevas infecciones indica que nos encontramos ante un éxito relativo de la prevención o si se prefiere ante un fracaso a medias. Alertados por esta situación, los investigadores psicosociales se han afanado por comprender los factores explicativos de los hábitos sexuales y de consumo de drogas. Al menos, hoy en día se tiene claro que la información no es un recurso suficiente para modificar el comportamiento humano. Resulta necesario también trabajar con las actitudes incrementando fundamentalmente la percepción de riesgo de la población general, entrenar en comportamientos saludables como el uso del preservativo o la desinfección de jeringas, ayudar a desarrollar la asertividad para negociar con la pareja la seguridad en las relaciones sexuales y mejorar la accesibilidad a los medios preventivos. Además, es necesario incrementar los esfuerzos que los gobiernos dirigen a la prevención, adelantar las intervenciones preventivas a edades más tempranas de lo que se hace actualmente, atender tanto a la población general como a las peculiaridades de colectivos concretos y tener muy en cuenta las variables culturales tanto al intentar exportar programas preventivos a otros países como en su aplicación a los colectivos de inmigrantes.

Por lo que respecta a la investigación biomédica y los avances científicos para la comprensión y el tratamiento de la enfermedad hay que decir que éstos han sido enormes. Si se tardó aproximadamente unos 200 años en conocer una enfermedad tan frecuente y grave como la sífilis, en apenas 20 años hemos aprendido cómo actúa el VIH dentro de nuestro organismo debilitándolo, cómo afecta a nuestro siste- 
ma inmunitario y en especial a los linfocitos TCD4, qué es lo que facilita que se replique dentro de nuestro cuerpo y además se han desarrollado fármacos nuevos de gran eficacia. La Terapia Antirretroviral Altamente Activa (HAART) que combina la acción de los Inhibidores de la Transcriptasa Inversa y los Inhibidores de la Proteasa, ha demostrado su capacidad para suprimir la carga viral de los pacientes hasta niveles indetectables, incrementar los indicadores de funcionamiento inmunitario tales como el recuento de linfocitos CD4, mejorar el estado clínico de los pacientes y disminuir su mortalidad, hasta tal punto que es esta terapia la que ha permitido que hoy podamos hablar del SIDA como una enfermedad no necesariamente fatal, sino crónica. De hecho los interrogantes tanto biomédicos como psicosociales que hoy plantea el SIDA se parecen mucho más a los de una enfermedad crónica que a los de una enfermedad terminal. Sin embargo, los nuevos y potentes tratamientos para el SIDA se enfrentan en la actualidad a dos graves problemas. El primero y quizás el más grave es que su altísimo coste deja al margen al $95 \%$ de los casos que se sitúan en el mundo subdesarrollado o en vías de desarrollo por motivos económicos. El segundo problema es que los actuales tratamientos conllevan un gran número de pastillas a ingerir diariamente en pautas complejas; se trata de una terapia de larga duración, quizás indefinida; y suele llevar aparejados efectos secundarios de cierta importancia; todo lo cual ayuda a explicar la frecuencia con que los pacientes no se adhieren adecuadamente al tratamiento, problemática que atenderemos más adelante (Ballester, 2002).

PROBLEMAS EMOCIONALES EN LAS PERSONAS CON INFECCIÓN POR VIH

En general, los estudios que exploran el estado psicológico de los pacientes con infección por VIH señalan un índice de morbilidad psicopatológica que oscila entre el $30 \%$ y el $80 \%$ de los afectados. En estudios con seropositivos norteamericanos prevalecen en primer lugar, los trastornos afectivos seguidos de los síndromes demenciales y los trastornos psicóticos, mientras que en trabajos que exploran la presencia de psicopatología en enfermos españoles predominan el trastorno por abuso de sustancias, los trastornos orgánicos mentales, trastornos adaptativos, ideación suicida y trastornos afectivos (Edo, 2003). En la revisión de la literatura realizada por Ballester (1999) se destacan los siguientes problemas psicológicos como habituales en los pacientes con VIH: baja autoestima, miedo a perder el atractivo físico e hipocondría, somatización y síntomas obsesivo-compulsivos, reaparición de conflictos sobre la orientación homosexual, disminución de la concentración y pérdida de memoria, fobias específicas, trastorno de personalidad antisocial y deseo sexual hipoactivo, abuso de alcohol y otras drogas, problemas de sueño y uso excesivo de sedantes.

La presencia de problemas emocionales en las personas con VIH/SIDA viene explicada, de entrada, por la psicopatología previa de los afectados, en segundo lugar por la dificultad para adaptarse a una enfermedad que impone importantes restricciones en la vida del enfermo, en tercer lugar como consecuencia de la misma enfermedad y de su tratamiento y en cuarto lugar, como efecto del estigma social asociado al SIDA.

Por lo que respecta a la morbilidad psicopatológica previa a la enfermedad hay que destacar que el trastorno por consumo de sustancias aparece, como es lógico, en la historia presente o pasada de los infectados por vía parenteral (Stoskopf, Kim y Glover, 2001). En la población española la incidencia de trastorno por dependencia de sustancias es de un $\mathbf{4 1 \%}$ 
(Ayuso, 1997). Además, este diagnóstico presenta a su vez una alta comorbilidad con otros como los trastornos de personalidad. De hecho, los pacientes con VIH presentan una alta prevalencia de trastornos de personalidad que llega a ser del $36 \%$ (Turrina et al., 2001). También en población homosexual se detecta una elevada tasa de trastornos mentales previa al diagnóstico (Perkins et al., 1994).

La adaptación a una enfermedad que hasta hace muy poco era considerada necesariamente mortal y todavía en la actualidad muy estigmatizada, supone un proceso largo en el cual pueden surgir múltiples manifestaciones psicopatologicas. Cuando las manifestaciones ante el shock inicial que supone el diagnóstico se prolongan en el tiempo o son muy intensas pueden llegar a desencadenar un trastorno adaptativo cuya gravedad vendrá determinada por factores como la historia previa de patología mental, su respuesta de afrontamiento a la enfermedad, el deterioro que produzca la enfermedad, el apoyo social, laboral y familiar, la disponibilidad de ayuda psicológica, etc. De entre todos los trastornos, parece que los cuadros afectivos mixtos, con sintomatología ansioso-depresiva son los más habituales (Weinrich, Atkinson, McCutchan y Grant, 1995). Especialmente frecuente es la ansiedad ante la muerte conforme la enfermedad va avanzando, que llega a ser experimentada por el $85 \%$ de los pacientes (Catania, Turner, Choi y Coates, 1992). Otra importante alteración psicológica a considerar es la ideación y los intentos de suicidio. La literatura indica que el riesgo de suicidio es uno de los principales síntomas psicopatológicos de las personas afectadas por el VIH (Roy, 2003). Su incidencia es superior a la del resto de la población, llegándose a señalar en algunos estudios que el riesgo de suicidio es veinte veces superior a los de la población general en el mismo segmento de edad (Kirchner, 1995). No obs- tante, resulta difícil atribuir esta alta tasa de suicidios al hecho de padecer la enfermedad. Algunos estudios revelan que la incidencia del suicidio ante el diagnóstico no es mucho mayor de la que se encuentra ante otras enfermedades como el cáncer y otros indican que en cualquier caso, un buen porcentaje de los pacientes con ideación suicida ya la tenían antes de infectarse con el VIH (ODowd, Biderman y Mc Kegney, 1993).

Los denominados factores yatrogénicos o efectos secundarios ocasionados por las terapias antirretrovirales y/o los tratamientos específicos para las infecciones oportunistas también pueden influir en el estado mental de estos pacientes. Así, por ejemplo, la ingesta de Zidovudina, uno de los antirretrovirales más habituales, puede producir astenia, ansiedad, depresión, estado de confusión, inquietud, manía y otros estados psicológicos en los pacientes (Edo, 2003). Además el VIH es un virus neurotrópico lo que significa que puede afectar al sistema nervioso central y provocar cuadros neurológicos en aproximadamente el $30 \%$ de los pacientes destacando entre otras complicaciones las encefalopatías, demencias y delirium (Ballester, 1999).

Finalmente, otra fuente de problemas emocionales en las personas afectadas es la que tiene que ver con las repercusiones sociales de la enfermedad. Las posibles consecuencias sociales que conlleva declarar que se es portador del VIH o enfermo de SIDA pueden incluir el rechazo, la estigmatización, marginación, culpabilización, etc., tanto desde el entorno laboral y social como desde los ámbitos más próximos al afectado como la familia, la pareja y los amigos. En un estudio reciente llevado a cabo en nuestro país (Edo, 2003) se encontró que los pacientes con infección por VIH percibían menor apoyo social que un grupo control de personas sanas y otro de pacientes oncológicos en los ámbitos social, 
laboral, familiar, de amistades y general. Por su parte, Lee, Kochman y Sikkema (2002) obtuvieron evidencia de que la mayoría de los seropositivos presentan una internalización del estigma asociado a su estatus como enfermo, internalización que lleva a estar más preocupados por la posibilidad de infectar a otras personas y a experimentar mayor ansiedad, depresión y desesperanza. Además, hay que recordar que el estigma de padecer SIDA en muchas ocasiones viene a sumarse a otros estigmas previos como el de ser gay o lesbiana (Yarhouse, 2003).

En definitiva, son muchos los motivos por los que los pacientes con VIH/SIDA suelen presentar problemas emocionales, problemas que no sólo amenazan su bienestar psicológico, sino también su propia supervivencia, como veremos a continuación.

\section{REPERCUSIÓN DE LOS FACTORES PSICOLÓGICOS EN LA PROGRESIÓN DE LA ENFERMEDAD}

Como hemos señalado, el hecho de que los pacientes con infección por VIH experimenten con frecuencia problemas psicológicos no sólo lleva a mermar de manera muy importante su calidad de vida a través del incremento de su malestar emocional. Los problemas emocionales también tienen su efecto sobre el estado físico de los pacientes y su propia supervivencia. Esta repercusión negativa sobre el estado físico de los enfermos se expresa fundamentalmente de tres modos. En primer lugar, a través de la relación entre los factores cognitivos y emocionales y la función inmune, cuestión que trataremos en este epígrafe. En segundo lugar, a través de su influencia sobre la adhesión al tratamiento antirretroviral, lo que analizaremos en el siguiente epígrafe. $Y$ en tercer lugar, por su relación con los hábitos de salud de los enfermos, hábitos que resultan fundamentales en la progresión de la enfermedad y que incluyen, desde el consumo de sustancias tóxicas hasta la nutrición o la prevención de reinfecciones.

En el año 1964, Solomon y Moods, al especular con una teoría que integraba las emociones, la respuesta inmune y el estado de salud (la enfermedad), ponían la semilla que más tarde sería abonada con los trabajos de Ader y Cohen sobre la aversión condicionada, dando como fruto la Psiconeuroinmunología. En el ámbito de la infección por VIH, una serie de estudios (Kemeny, 2003) demuestran la gran importancia de la interacción de variables psicológicas, endocrinas e inmunitarias en el pronóstico y la evolución de la enfermedad, sugiriendo que determinados factores psicológicos pueden potencialmente afectar al curso de la infección, tanto respecto a la aparición de problemas físicos y/o emocionales como a la respuesta inmunitaria o a la propia supervivencia. Ulla y Remor (2002) señalan cómo tanto los aspectos comportamentales (estilos de vida) como los psicológicos (control del estrés y estrategias de afrontamiento) y sociales (apoyo social) pueden influir de manera importante en la progresión de la infección. Recientemente han sido destacados otros factores psicológicos en su influencia sobre la función inmune como por ejemplo: la depresión sobre la actividad de las células NK (natural killers), los linfocitos TCD8 , la carga viral y la mortalidad de las enfermas con VIH (Evans et al., 2002); la expresión emocional sobre la supervivencia a largo plazo de los enfermos ( $\mathrm{O}^{\prime} \mathrm{Cleirigh}$ et al., 2003); el estrés vital como factor independiente sobre el riesgo de neoplasias cervicales en mujeres con VIH (Pereira et al., 2003); e incluso se ha tratado la influencia indirecta que sobre la función inmune pue- 
dan ejercer aspectos como las creencias religiosas y espirituales de los individuos (Ironson y Schneiderman, 2002).

Asimismo, determinadas intervenciones psicológicas dirigidas a mejorar la calidad de vida de los enfermos y a controlar sus problemas emocionales han conseguido reducir las enfermedades oportunistas e incrementar el número de linfocitos TCD4. En este sentido, es conocido el magnífico trabajo de KiecoltGlaser y Glaser (1992) en el que estos autores realizan una revisión de la literatura científica sobre la modulación de la función inmune a través de tratamientos psicológicos. En este artículo, los autores recogen evidencias de la eficacia de intervenciones consistentes en incrementar estrategias como la relajación, hipnosis, ejercicio físico, autorrevelación, búsqueda de apoyo social, autoeficacia y también de programas cognitivocomportamentales multicomponentes ante el VIH como el de Antoni et al. (1991). Más recientemente, Robinson (2002) ha informado de los efectos inmunitarios de un programa estructurado de ocho sesiones en el que se entrenaba a los enfermos a enfrentarse a los estresores que impone la enfermedad y a otros estresores vitales. Tras el programa, se observaba una mejora global de la salud y una mayor actividad de las células NK en los pacientes.

No obstante, es necesario seguir trabajando en este ámbito ya que la relación entre los aspectos emocionales y psicológicos en general y la función inmune en los pacientes con VIH es compleja. Un ejemplo de esta complejidad lo constituye el reciente trabajo de Motivala et al. (2003) revelando que el malestar psicológico se encuentra relacionado con un menor número de linfocitos $\mathrm{T}$ y de células $B$ (productoras de anticuerpos) en pacientes con VIH, pero que esta relación sólo se da en aquéllos con baja carga viral.

\section{LA ADHESIÓN AL TRATAMIENTO: UNA CUESTIÓN CRÍTICA}

Como se ha indicado anteriormente el desarrollo de la Terapia Antirretroviral Altamente Activa (HAART) ha permitido comenzar a hablar del SIDA como de una enfermedad crónica, en la que tanto la calidad de vida como la supervivencia de los enfermos poco tienen que ver con la situación existente apenas hace unos años.

Sin embargo, numerosos estudios (Ballester, Reinoso, García y Campos, 2000; Murphy, Wilson, Durako, Muenz y Belzer, 2001) han denunciado lo frecuente que resultan los problemas de adhesión al tratamiento antirretroviral (para una revisión extensa sobre esta tema, consultar el trabajo de Ballester, 2002).

Cuando hablamos de adhesión farmacológica, es decir, hasta qué punto los pacientes toman los fármacos prescritos, la mayoría de investigaciones informan de un $50 \%$ de pacientes que no siguen adecuadamente el tratamiento (Witteveen y van Ameijden, 2002). Pero la adhesión se debe referir también a aspectos como la adopción de medidas preventivas de reinfecciones y la asistencia al régimen de visitas médicas. En este sentido, Ballester et al. (2000) han encontrado que un $53 \%$ de los pacientes con infección por VIH dejan de tomar en ocasiones los fármacos prescritos, el $14 \%$ sigue realizando las mismas prácticas de riesgo que les llevó a la infección por VIH y el $10 \%$ no cumple con el régimen de visitas. Fuera de nuestras fronteras, Gwadz et al. (1999) encontraron en su estudio que el porcentaje de no adhesión era del $45 \%$ de los pacientes. Y más recientemente Murphy, Wilson, Durako, Muenz y Belzer (2001) hallaron que tan sólo el $41 \%$ de adolescentes con VIH presentaban una adhesión plena al tratamiento. 
Por lo tanto, sabemos que la falta de adhesión a los tratamientos con antirretrovirales es muy frecuente. A eso hay que añadir la gravedad que en el tratamiento del SIDA incorpora una adhesión inadecuada. La investigación muestra que incluso una leve falta de adhesión reduce de forma importante los beneficios del tratamiento a través del incremento de la carga viral, la mutación del VIH y su resistencia a los fármacos, la cual puede transmitirse a otros individuos (Chesney, 2003).

La gravedad de los problemas de adhesión al tratamiento en los pacientes con infección por VIH ha hecho que se investiguen los posibles factores relacionados con ellos y su efecto modulador sobre la adhesión. Algunos factores no han mostrado hasta ahora ser excesivamente relevantes, como es el caso de la edad, el sexo o la raza (Ferguson et al., 2002). Sin embargo, distintos estudios han concluido la relevancia que pueden tener algunas variables que presentamos en el cuadro 1. En este trabajo sólo referiremos los estudios más recientes (para una revisión de los estudios previos es posible consultar el trabajo de Ballester, 2002). Así, entre los factores que se han visto relacionados con la adhesión se encuentran el nivel educativo, el grado de información respecto a la enfermedad, las creencias culturales del paciente, la valoración de la gravedad de la enfermedad, la percepción acerca de la eficacia del tratamiento, la complejidad del tratamiento, la vulnerabilidad percibi$\mathrm{da}$, la intolerancia a los efectos secundarios, la creencia acerca de la toxicidad de los fármacos, la relación con el facultativo, la inestabilidad en las vidas de los pacientes (p.ej., no tener un hogar), el ánimo depresivo, la ansiedad, los problemas psicopatológicos en general, la calidad de vida, la falta de apoyo social, el Sentido de Coherencia, el consumo excesivo de alcohol, la historia de consumo de drogas o simplemente el olvido.
A pesar de todos los estudios mencionados los problemas de adherencia al tratamiento de los pacientes con VIH son tan frecuentes y tan complejos que ninguna combinación de variables sociodemográficas y psicológicas ha sido capaz de predecir en un alto grado qué pacientes no van a seguir las prescripciones médicas. Por otro lado, el número de correlatos de la adhesión a los tratamientos antirretrovirales propuesto por diferentes autores hasta el momento es muy alto. En la revisión de la literatura sobre el tema realizada por Fogarty et al. (2002) se identificaron más de 200 variables distintas. $\mathrm{Y}$ por si fuera poco, la metodología utilizada en los diferentes estudios es tan diferente como la propia definición de adherencia adoptada (en unos estudios se consideran adherentes a los que cumplen al $\mathbf{1 0 0} \%$ con la medicación, en otros a los que la siguen en un $80 \% . .$.$) , el modo de evaluar la adhesión$ (autoinforme del paciente, valoración del médico, monitorización electrónica y resultados clínicos fundamentalmente) y las características de los pacientes considerados (en cuanto a edad, sexo, cultura, consumo de drogas, orientación sexual...). Todo ello dificulta llegar a conclusiones generalizables. Seguramente, tal como señalan Remien et al. (2003), deberíamos considerar la adhesión como un fenómeno dinámico que cambia en el tiempo, acompañando el cambio de creencias, actitudes, emociones y eventos vitales cotidianos o trascendentes en la vida del paciente. Lo que parece claro es que estamos hablando de un problema cuya solución es de vital importancia dada la existencia de tratamientos de gran eficacia que pueden ayudar a incrementar no sólo la esperanza de vida del paciente, sino también su calidad de vida. De esto se deriva la relevancia de desarrollar programas eficaces para mejorar la adhesión de los seropositivos. 
Cuadro 1. Revisión de estudios acerca de los factores relacionados con la adhesión en pacientes VIH+

Factores relacionados con la adhesión en pacientes con infección por VIH Estudios

Nivel educativo

Informacion sobre la enfermedad

Catz, Heckman, Kochman y DiMarco (2001)

Creencias culturales del paciente

Weiss et al. (2003)

Valoración de la gravedad de la enfermedad

Siegel, Karus y Schrimshaw (2000)

Ballester et al. (2000)

Gao, Nau, Rosenbluth, Scott y Woodward (2000)

Percepción acerca de la eficacia del tratamiento

Ballester et al. (2000)

Complejidad del tratamiento

Murphy, Johnston y Martin (2000)

Vulnerabilidad percibida

Gao et al. (2000)

Intolerancia a los efectos secundarios

Catz et al. (2000)

Creencia acerca de la toxicidad de los fármacos

Smith, Rapkin, Morrison y Kammerman (1997)

Relación con el facultativo

Ballester et al. (2000)

Murphy et al. (2000)

Catz et al. (2001)

Demmer (2003)

Inestabilidad en las vidas de los pacientes

(p.e. no tener un hogar)

Ánimo depresivo

Bangsburg, Tulsky, Hecht y Moss (1997)

Murphy et al. (2000)

Catz et al. (2000)

Murphy et al. (2001)

Remor (2002)

Ansiedad

Catz et al. (2001)

Remor (2002)

Problemas psicopatológicos en general

Sternhell y Corr (2002)

Calidad de vida

Penedo et al. (2003)

Sentido de coherencia

Cederfjaell et al. (2002)

Falta de apoyo social

Catz et al. (2000)

Remor (2002)

Cox (2002)

Power et al. (2003)

Consumo excesivo de alcohol

Catz et al. (2001)

Power et al. (2003)

Historia de consumo de drogas

Arnsten et al. (2002)

Power et al. (2003)

Ballester et al. (2003)

Olvido

Chesney et al. (2000)

INTERVENCIÓN PSICOLOGICA ANTE LA INFECCIÓN POR VIH

Dada la importancia que tiene la adhesión al tratamiento en los pacientes con VIH/SIDA algunas de las intervenciones propuestas tienen como fin mejorar esta adhesión. De acuerdo con la complejidad que encierra la adhesión y la multitud de factores implicados, los programas son en muchas ocasiones interdisciplinares, con la participación de psicólogos pero también de médicos, enfermeros, farmacéuticos y trabajadores sociales. Algunos 
se han dirigido a intervenir sobre el sistema sanitario creando unidades de atención multidisciplinar para mejorar la accesibilidad de los pacientes a los cuidados. Otros programas se han centrado más en las habilidades de comunicación de los profesionales sanitarios. Finalmente, otros se han dirigido a aspectos relacionados con el paciente como el ajuste del tratamiento a su funcionamiento cotidiano, entrenamiento en solución de problemas, enseñarles a superar las barreras potenciales, refuerzo por sus esfuerzos para seguir el tratamiento y uso de tecnologías para seguir las pautas del tratamiento, tales como los pastilleros o "avisadores» electrónicos (para una revisión ver el trabajo de Ballester, 2002).

Sin embargo, algunos estudios bastante recientes (Sternhell y Corr, 2002; Remor, 2002; Halkitis, Parsons, Wolitski y Remien, 2003; Penedo et al., 2003), dan una importancia especial a la existencia de problemas emocionales como factor de riesgo para una baja adhesión terapéutica, señalando que el tratamiento de éstos debería ser una prioridad. De esta constatación se deriva que recientemente se hayan desarrollado programas que más que estar exclusivamente orientados a la promoción de la adhesión, pretenden mejorar la calidad de vida general del enfermo, potenciando especialmente los aspectos emocionales, si bien muy pocos ofrecen datos de su eficacia (Fogarty et al., 2002).

Como se puede apreciar en el cuadro 2, uno de los primeros estudios que cabe citar es el de Coates y McKusick (1987) quienes hallaron que tras la aplicación de un programa grupal de entrenamiento en técnicas de manejo del estrés, los pacientes con VIH mostraban menor estrés, depresión y menor frecuencia en las conductas de riesgo que los pacientes del grupo control. En un trabajo publicado por estos autores dos años más tarde (Coates, McKusick, Kuno y Stites, 1989), describían una terapia de grupo que consistía en ocho sesiones de dos horas de duración cada una en las que se enfatizaba el entrenamiento en relajación, el cambio de hábitos de salud como la reducción del consumo de tabaco y alcohol y el incremento del descanso y del ejercicio físico, así como otras habilidades para el manejo del estrés. El resultado fue la mejoría en distintas variables clínicas relacionadas con el estrés y el estado emocional de los pacientes.

Emmot (1991), por su parte, evaluó la eficacia de un programa de intervención grupal para pacientes con VIH encontrando que tras el programa había un descenso del ánimo depresivo y de la ansiedad. También Folkman et al. (1991) encontraron una mejora en los estilos de afrontamiento, menor autoinculpación y menor depresión tras una terapia grupal aplicada a hombres homosexuales.

Algunos autores se han planteado el desarrollo de programas aplicables ya no a los pacientes que llevan tiempo afectados por la enfermedad, sino también a aquellos pacientes que acaban de recibir el impacto de su diagnóstico. Es el caso de Antoni et al. (1991) que aplicaron un programa cognitivo-comportamental a hombres homosexuales antes de la notificación del resultado de las pruebas de detección de anticuerpos. El programa incluía técnicas para el manejo del estrés, entrenamiento en relajación, habilidades de afrontamiento de estresores, incremento de la autoeficacia y aumento de las relaciones sociales. Los resultados evidenciaron que los pacientes tratados mostraban menor depresión y mayor ajuste y sensación de control tras el diagnóstico.

Por su parte, Kelly et al. (1993) evaluaron la eficacia de un programa grupal para pacientes seropositivos con depresión. Asignaron aleatoriamente 68 hombres seropositivos con una alta puntuación en depresión a uno de tres grupos 
Cuadro 2. Revisión de los componentes terapéuticos de algunos programas de intervención para pacientes con infección por VIH/SIDA

Autores
Coates y McKusick (1987)
Coates, McKusick, Kuno
y Stites (1989)

Antoni et al. (1991)

Tratamiento

Programa grupal de entrenamiento en técnicas de manejo del estrés.

Terapia de grupo (ocho sesiones de dos horas de duración cada una) en las que se enfatizaba el entrenamiento en relajación, el cambio de hábitos de salud como la reducción del consumo de tabaco y alcohol y el incremento del descanso y del ejercicio físico, así como otras habilidades para el manejo del estrés.

Técnicas para el manejo del estrés, entrenamiento en relajación, habilidades de afrontamiento de estresores ambientales, valoración del estrés, afrontamiento activo, incremento de la autoeficacia y aumento de las relaciones sociales.

Kelly et al. (1993)

Terapia grupal cognitivo-conductual que inclufa estrategias para reducir la ansiedad y la depresión como la reestructuración cognitiva, la relajación muscular, la autorrevelación sobre la enfermedad, la adopción de medidas de sexo seguro y el establecimiento de una red social de apoyo.

Eller (1995)

Lutgendorf et al. (1997)

Aplicación individual de la relajación muscular y la imaginación guiada a través de grabaciones.

Terapia (10 sesiones de 90 min. de duración) en la que se entrenaba a pacientes homosexuales en técnicas de manejo del estrés

McPherson et al. (2000)

Técnicas de consejo breve dirigidas específicamente a la mejora de la adherencia al tratamiento antirretroviral

Molassiotis, Callaghan, Twinn, Lam, Chung y Li (2002)

12 sesiones de intervención grupal que incluyen el entrenamiento de estrategias para reducir la ansiedad, la depresión y mejorar la calidad de vida de los pacientes.

Pomeroy, Green y

Van-Laningham (2002).

Programa grupal dirigido a parejas

heterosexuales serodiscordantes que incluye un componente educativo en el que se da información acerca de la enfermedad; un segundo componente de apoyo que incluye estrategias cognitivas y de solución de problemas; y un tercer componente que incluye el desarrollo de estrategias de afrontamiento, animar a los pacientes para la realización de actividades gratificantes y el incremento de actividades sociales.

Smith, Rublein, Marcus, Brock y Chesney (2003)

Programa de automanejo para mejorar la adhesión terapéutica que incluye el desarrollo de habilidades implicadas en la toma de medicación, tres visitas semanales para consultas relacionadas con la misma y el feedback mensual de la toma de medicación utilizando monitores electrónicos en los botes de fármacos.

Ballester (2003)

Programa de tratamiento grupal que consta de ocho sesiones que incluyen un componente educativo de información al paciente, estrategias cognitivas, entrenamiento en autoestima, relajación, solución de problemas, estrategias para el autocuidado y prevención de recaídas.

experimentales. El primero consistía en un tratamiento grupal cognitivo-conductual que incluía estrategias para reducir la ansiedad y la depresión como la reestruc- turación cognitiva, la relajación muscular, la autorrevelación sobre la enfermedad, la adopción de medidas de sexo seguro y el establecimiento de una red social de apo- 
yo; el segundo era un grupo de apoyo; y el tercero consistía en psicoterapia individual. Los resultados mostraron que ambos tratamientos grupales producían una mejoría en los pacientes significativamente mayor que la terapia individual.

Dos años después, Eller (1995) evaluó la eficacia de dos técnicas de reducción del estrés en pacientes seropositivos: la relajación muscular y la imaginación guiada. La principal novedad era la brevedad del tratamiento en ambos casos: 6 semanas en las que únicamente se utilizaba una grabación de 21 minutos de imaginación guiada o una grabación de 12 minutos de relajación. Sin embargo, ambos tratamientos mejoraron a los pacientes más que la condición control en que no se aplicaba ninguna técnica.

Más extenso era el tratamiento de Lutgendorf et al. (1997) con una duración de 10 sesiones de 90 minutos en las que se entrenaba a pacientes homosexuales en técnicas de manejo del estrés. Los resultados indicaron que los pacientes que habían recibido el tratamiento, en comparación con el grupo control, experimentaban una reducción de la ansiedad y la disforia, además de mejorar su sistema inmunitario.

De nuevo, el estudio de McPherson et al. (2000), se dirigió a evaluar la eficacia de técnicas de consejo breve, pero en este caso el fin del programa era específicamente a la mejora de la adhesión al tratamiento antirretroviral, obteniendo una mayor adhesión terapéutica, menos infecciones oportunistas y menos ingresos entre los que recibieron la intervención, en comparación con un grupo control. Recientemente, Smith, Rublein, Marcus, Brock y Chesney (2003) también han aportado datos positivos acerca de la eficacia comparada con un grupo control de un programa de automanejo para mejorar la adhesión terapéutica que incluye el desarrollo de habilidades implicadas en la toma de medicación, tres visitas semanales para consultas relacionadas con la misma y el feedback mensual de la toma de medicación utilizando monitores electrónicos.

Frente a estos dos últimos programas, centrados en la mejora de la adhesión terapéutica, el programa cognitivo-comportamental de Molassiotis et al. (2002) consiste en 12 sesiones de intervención grupal que incluyen el entrenamiento de estrategias para reducir la ansiedad, la depresión y mejorar la calidad de vida de los pacientes. Los resultados mostrados por los autores evidencian que los pacientes que recibieron este tratamiento mejoraron en términos de tensión ansiosa, depresión, estado de confusión y estado general de ánimo comparados con los pacientes del grupo control.

Finalmente, en nuestro país, el programa de tratamiento grupal propuesto por Ballester (2003) consta de ocho sesiones que incluyen un componente educativo de información al paciente, estrategias cognitivas, entrenamiento en autoestima, relajación, solución de problemas, estrategias para el autocuidado (adhesión al tratamiento, hábitos de salud y prevención de reinfecciones) y un último módulo de prevención de recaídas. En sus resultados preliminares, se han observado cambios significativos tras el tratamiento en el grado de adhesión de los pacientes, variables clínicas relevantes como la ansiedad, depresión o la autoestima y en otros aspectos como el grado de hipocondría de los pacientes, su perturbación afectiva, irritabilidad, grado de interferencia de la enfermedad, apoyo social percibido y locus de control sobre la salud.

\section{RETOS ACTUALES EN LA INVESTIGACIÓN PSICOLÓGICA SOBRE EL SIDA}

Si el reto para la investigación biomédica en torno al SIDA es fundamentalmen- 
te el desarrollo de nuevos fármacos sin las limitaciones de los actuales y de una vacuna que pueda proteger al mayor porcentaje posible de la población, el reto para la investigación psicológica no es menos importante.

Probablemente pocas cosas sean tan complejas, difíciles de explicar y también de modificar, como el comportamiento humano. Desde un punto de vista idealista y del deseo, podríamos decir que la lucha contra el SIDA debería ser sencilla. Dado que el VIH se transmite a través de unos pocos comportamientos de riesgo bien identificados, bastaría con informar a la población acerca de la necesidad de no realizar esos comportamientos. En el caso de que en un porcentaje mínimo de situaciones la prevención fallara, los pacientes infectados podrían beneficiarse de los actuales tratamientos farmacológicos altamente efectivos que les podrían garantizar una cierta calidad de vida y una larga supervivencia.

Sin embargo, la realidad es bien distinta. Nos encontramos con que la mayoría de los seres humanos realizan conductas de riesgo para la transmisión del VIH, a pesar de que puedan tener información respecto a las vías de contagio. Pero además, de ese porcentaje mayoritario, muy pocos acuden a realizarse las pruebas de detección de anticuerpos. Por si fuera poco, de entre los que acuden, muchos no vuelven para conocer los resultados de las pruebas (Johnson et al., 2003). Una vez conocidos los resultados, cuando son indicadores de ausencia de infección, muchos individuos reinciden en las conductas de riesgo. Cuando son indicadores de presencia de infección, un alto porcentaje de pacientes llevan a cabo conductas que les somete a reinfecciones y se adhieren escasamente al tratamiento que les podría prolongar y mejorar su vida.

La investigación psicológica futura debería centrarse en todos estos ámbitos del comportamiento que enfrenta a muchas personas a una situación dramática. En el ámbito de la prevención, es necesario aunar los esfuerzos para mejorar nuestra comprensión de los factores implicados en las conductas de riesgo con el fin de desarrollar intervenciones preventivas que hayan demostrado su eficacia, a edades más tempranas y de forma que lleguen a todos los segmentos de la población. En el ámbito de la atención a las personas que ya padecen la enfermedad, aspecto en el que nos hemos centrado en este artículo, es necesario que nos demos cuenta de que las necesidades psicosociales de los enfermos están cambiando y se parecen más a las de un enfermo crónico que a las de un enfermo terminal.

En este sentido, se nos plantean nuevos retos como por ejemplo la importancia de estudiar qué es lo que sucede con los niños y adolescentes infectados cuando crecen y llegan a adultos. Los datos nos indican que los jóvenes afectados por el VIH suelen, con mayor probabilidad que los no infectados, no haber finalizado sus estudios, haber perdido a un padre y que aquéllos que tienen un menor apoyo social y que no pueden hablar con otros significativos acerca de los problemas asociados a su enfermedad, padecen con mayor frecuencia depresión y otros problemas emocionales (Battles y Wiener, 2002). Las escuelas podrían cumplir una función importante proporcionando estabilidad a los estudiantes cuyos familiares están enfermos o han muerto por el SIDA (Duggan, 2003). En este segmento de la población (niños y adolescentes), también se requiere de mayor investigación acerca de los factores asociados a su adhesión al tratamiento (Steele y Grauer, 2003).

Por lo que respecta a la intervención psicológica, observamos nuevos retos para el futuro. En primer lugar, es necesario que se realicen más estudios acerca 
de la eficacia de las intervenciones. Precisamos conocer qué componentes terapéuticos son los más activos en la mejoría de los pacientes y cuáles son los factores moduladores de esta mejoría. En segundo lugar, habría que investigar algunos aspectos que tienen más que ver con la forma de aplicar los programas que con los contenidos en sí mismos.

Uno de ellos hace referencia al tratamiento de las parejas serodiscordantes. Dado que el malestar emocional en estas parejas guarda una estrecha relación con la satisfacción diádica, la satisfacción sexual, la autoinculpación y el apoyo de la propia pareja, sería necesario que las intervenciones clínicas para estas parejas se centraran en la relación y no en el enfermo por separado (Remien, Wagner, Dolezal y Carballo, 2003). De hecho ya disponemos de datos que hablan de la eficacia de programas como el de Pomeroy, Green y Van-Laningham (2002) que parten de esta idea.

Otra necesidad que comienza a revelarse es la de implantar programas de intervención psicológica dirigidos a los cuidadores de los niños con infección por VIH para ayudarles a enfrentarse al estrés que supone su cuidado (Chalfin, Grus y Tomaszeski, 2002).

Respecto a las personas mayores que llevan años padeciendo la enfermedad, algunos autores indican que en los programas para estos pacientes debería disminuirse la atención a lo patológico y al estigma e intentar que los pacientes se centren en áreas de su vida en las que están funcionando adecuadamente con el fin de incrementar la sensación de control de su vida (Orsulic-Jeras, Shepherd y Britton, 2003).

Otra línea futura de trabajo se refiere a la investigación hacia formas más breves de intervención. Un ejemplo es el trabajo de Hershberger, Wood y Fisher (2003) en el que se compara la eficacia de un programa de 9 sesiones a lo largo de cuatro meses para reducir las conductas de riesgo entre usuarios de drogas intravenosas con la de un programa consistente simplemente en dos sesiones de counseling, no evidenciándose diferencias significativas. $Y$ también el trabajo de Paterson, Shaw y Semple (2003) que aporta datos acerca de la eficacia de una intervención tan breve como la que pueda aplicarse en una sola sesión para reducir las conductas de riesgo para la transmisión del VIH entre los pacientes seropositivos. Este tipo de intervenciones breves pueden ser muy importantes para poder atender ciertas necesidades clínicas. Así, algunos estudios recientes revelan que aproximadamente un $\mathbf{5 8 \%}$ de los pacientes que son diagnosticados como seropositivos no vuelven al centro sanitario para conocer el resultados de las pruebas y recibir consejo (Hightow et al., 2003). Por lo tanto, sería útil implantar programas de intervención breve en el mismo momento de realización de las pruebas diagnósticas para conseguir que las personas que acuden a hacerse estas pruebas vuelvan a recoger los resultados.

Finalmente, cada vez más autores defienden el papel que pueden jugar las nuevas tecnologías como Internet no sólo para lanzar mensajes de prevención a la población general o a grupos específicos (Rietmeijer, 2003), sino también como una estrategia de ayuda a personas ya infectadas por el VIH (Kalichman et al., 2002).

\section{REFERENCIAS BIBLIOGRÁFICAS}

Antoni, M., Baggett, L., Ironson, G., LaPerriere, A., August, S., Klimas, N., Schneiderman, N., y Fletcher, M. (1991). Cognitivebehavioral stress management intervention buffers distress responses and immunologic changes following notification of HIV1 seropositivity. Journal of Consulting and Clinical Psychology, 59, 906-915. 
Arnsten, J., Demas, P., Grant, R., Gourevitch, M., Farzadegan, H., Howard, A., y Schoenbaum, E. (2002). Impact of active drug use on antiretroviral therapy adherence and viral suppression in HIV-infected drug users. Journal of General Internal Medicine, 17, 377-381.

Ayuso, J.L. (1997). Trastornos neuropsiquiátricos en el SIDA. Madrid: McGraw Hill Interamericana.

Ballester, R. (1999). SIDA: Prevención y Tratamiento Psicológico en la Infección por $V I H$ y SIDA. Madrid: UNED-Fundación Universidad Empresa.

Ballester, R. (2002). Adhesión terapéutica: Revisión histórica y estado de la cuestión en la Infección por VIH/SIDA. Revista de Psicopatología y Psicología Clínica, 3, 151175.

Ballester, R. (2003). Eficacia terapéutica de un programa de intervención grupal cognitivo-comportamental para mejorar la adhesión al tratamiento y el estado emocional de pacientes con infección por VIH/SIDA. Psicothema, 15, 517-523.

Ballester, R., Reinoso, I., Campos, A., y García, S. (2003). ¿Existe un patrón diferencial de conducta de enfermedad y adhesión al tratamiento de la infección por VIH/SIDA en pacientes con historia de abuso de drogas? Revista de Psicopatología y Psicología Clínica, 2, 127-138.

Ballester, R., Reinoso, I., García, S., y Campos, A. (2000). Adherencia al tratamiento en la infección por VIH. Análisis y Modificación de Conducta, 109, 689-718.

Bangsburg, D., Tulsky, J.P., Hecht, F.M., y Moss, A.R. (1997). Protease inhibitors in the Homeless. Journal of the American Medical Association, 278, 63-65.

Battles, H., y Wiener, L. (2002). From adolescence through young adulthood: Psychosocial adjustment associated with longterm survival of HIV. Journal of Adolescent Health, 30, 161-168.

Catania, J.A., Turner, H., Choi, K., y Coates, T. (1992). Coping with death anxiety: helpseeking and social support among gay men with various HIV diagnoses. AIDS, 6 , 999-1005.

Catz, S., Heckman, T., Kochman, A., y DiMarco, M. (2001). Rates and correlates of HIV treatment adherence among late middleaged and older adults living with HIV disease. Psychology, Health and Medicine, 6, 47-58.

Catz, S., Kelly, J., Bogart, L., Benotsch, E., y McAuliffe, T. (2000). Patterns, correlates and barriers to medication adherence among persons prescribed new treatments for HIV disease. Health Psychology, 19, 124-133.

Cederfjaell, C., Langius, E., Lidman, K., y Wredling, R. (2002). Self-reported adherence to antiretroviral treatment and degree of sense of coherence in a group of HIV-infected patients. AIDS Patient Care and STD's, 16, 609-616.

Chalfin, S., Grus, C., y Tomaszeski, L. (2002). Caregivers's stress secondary to raising young children with HIV infection: a preliminary investigation. Journal of Clinical Psychology in Medical Settings, 9, 211-218.

Chesney, M. (2003). Adherence to HAART regimens. AIDS Patient Care and STD's, 17, 169-177.

Chesney, M., Ickovics, J., Chambers, D., Gifford, A., Neidig, J., Zwickl, B., y Wu, A. (2000). Self-reported adherence to antiretroviral medications among participants in HIV clinical trials: The AACTG Adherence Instruments. AIDS Care, 12, 255-266.

Coates, T., y McKusick, L (1987, Junio). The efficacy of stress management in reducing high risk behavior and improving immune function in HIV antibody positive men. Paper presented at the Third International Conference on AIDS, Washington, D.C.

Coates, T., McKusick, L., Kuno, R., y Stites, D. (1989). Stress reduction training changed number of sexual partners but not immune function in men with HIV. American Journal of Public Health, 79, 885-886.

Cox, L. (2002). Social support, medication compliance and HIV/AIDS. Social Work in Health Care, 35, 425-460.

Demmer, C. (2003). Relationship with health care provider and adherence to HIV medications. Psychological Reports, 93, 494-496.

Duggan, D. (2003). Children and young people affected by AIDS. En S. Books, Invisible children in the society and its schools. Mahwah, N.J.: Lawrence Erlbaum Associates. 
Edo, M.T. (2003). Perfil psicosocial, estado emocional y conducta de enfermedad en pacientes con infección por VIH/SIDA. Tesis Doctoral. Universidad de Castellón.

Eller, L. (1995). Effects of two cognitive-behavioral interventions on immunity and symptoms in persons with HIV. Annals of Behavioral Medicine, 17, 339-348.

Emmott, S. (1991, Junio). Cognitive group therapy for coping with HIV Infection. Paper presented at the Seventh International Conference on AIDS, Florencia (Italia)

Evans, D., Ten-Have, T., Douglas, S., Gettes, D., Morrison, M., Chiappini, M., BrinkerSpence, P., Job, C., Mercer, D., Wang, Y., Cruess, D., Dube, B., Dalen, E., Brown, T., Bauer, R., y Petitto, J. (2002). Association of depression with viral load, CD8 T lymphocytes and natural killer cells in women with HIV infection. American Journal of Psychiatry, 159, 1752-1759.

Ferguson, T., Stewart, K., Funkhouser, E., Tolson, J., Westfall, A., y Saag, M. (2002). Patient perceived barriers to antiretroviral adherence: Associations with race. AIDS Care, 14, 607-617.

Fogarty, L., Roter, D., Larson, S., Burke, J., Gillespie, J., y Levy, R. (2002). Patient adherence to HIV medication regimens: a review of published and abstract reports. Patient Education and Counseling, 46, 93-108.

Folkman, S., Chesney, M., McKusick, L., Ironson, G., Johnson, D., y Coates, T. (1991). Translating coping theory into an intervention. En J. Eckenrode (Ed.), The Social Context of Stress. Nueva York: Plenum Press.

Gao, X., Nau, D., Rosenbluth, S., Scott, V., y Woodward, C. (2000). The relationship of disease severity, health beliefs and medication adherence among HIV patients. AIDS Care, 12, 387-398.

Gwadz, M., De-Vogli, R., Rotheram-Borus, M.J., Diaz, M., Cisek, T., James, N., y Tottenham, N. (1999). Behavioral practices regarding combination therapies for HIV/AIDS. Journal of Sex Education and Therapy, 1-2, 81-88.

Halkitis, P., Parsons, J., Wolitski, R., y Remien, R. (2003). Characteristics of HIV antiretroviral treatments, access and adhe- rence in an ethnically diverse sample of men who have sex with men. AIDS Care, 15, 89-102.

Hershberger, S., Wood, M., y Fisher, D. (2003). A cognitive-behavioral intervention to reduce HIV risk behaviors in crack and injection drug users. AIDS and Behavior, 7, 229-243.

Hightow, L., Miller, W., Leone, P., Wohl, D., Smurzynski, M., y Kaplan, A. (2003). Failure to return for HIV postest counseling in an STD clinic population. AIDS Education and Prevention, 15, 282-290.

Ironson, G., y Schneiderman, N. (2002). Psychosocial factors, spirituality/religiousness, and immune function in HIV/AIDS patients. En H. Koenig y H. Cohen (Ed.), The link between religion and health: Pyschoneuroimmunology and the faith factor (pp. 139-159). London: Oxford University Press.

Johnson, R., Martínez, J., Botwinick, G., Bell, D., Sell, R., Friedman, L., Dodds, S., Shaw, K., Siciliano, C., Walker, L., y Sotheran, J. (2003). What youth need: adapting HIV care models to meet the lifestyles and special needs of adolescents and young adults. Journal of Adolescent Health, 33 (supl.2), 4-9.

Kalichman, S., Weinhardt, L., Benotsch, E., DiFonzo, K., Luke, W., y Austin, J. (2002). Internet access and Internet use for health information among people living with HIVAIDS. Patient Education and Counseling, 46, 109-116.

Kelly, J., Murphy, D., Bahr, G., Kalichman, S., Morgan, M., Stevenson, L., Koob, J., Brasfield, T., y Bernstein, B. (1993). Outcome of cognitive-behavioral and support group brief therapies for depressed persons diagnosed with HIV infection. American Journal of Psychiatry, 150, 1679-1680.

Kemeny, M. (2003). An interdisciplinary research model to investigate psychosocial cofactors in disease: Application to HIV-1 pathogenesis. Brain, Behavior and Immunity, 17 (supl.), 62-72.

Kiecolt-Glaser, J., y Glaser, R. (1992). Psychoneuroimmunology: Can psychological interventions modulate immunity? Journal of Consulting and Clinical Psychology, 60, 1-7 
Kirchner, J. (1995). AIDS and suicide. Journal of Family Practice, 41, 493-496.

Lee, R., Kochman, A., y Sikkema, K. (2002). Internalized stigma among people living with HIV-AIDS. AIDS and Behavior, 6, 309-319.

Lutgendorf, S., Antoni, M., Ironson, G., Klimas, N., Kumar, M., Starr, K., McCabe, P., Cleven, K., Fletcher, M., y Schneiderman, N. (1997). Cognitive-behavioral stress management decreases dysphoric mood and herpes simplex virus-type 2 antibody titers in symptomatic HIV-seropositive gay men. Journal of Consulting and Clinical Psychology, 65, 31-43.

McPherson, S., Malow, R., Penedo, F., Jones, D., Schneiderman, N., y Klimas, N. (2000). Enhancing adherence to combination antiretroviral therapy in non-adherent HIVpositive men. AIDS Care, 12, 399-404.

Molassiotis, A., Callaghan, P., Twinn, S., Lam, S., Chung, W., y Li, C. (2002). A pilot study of the effects of cognitive-behavioral group therapy and peer support/counseling in decreasing psychological distress and improving quality of life in Chinese patients with symptomatic HIV disease. AIDS Patient Care and STD's, 16, 83-96.

Motivala, S., Hurwitz, B., Llabre, M., Klimas, N., Fletcher, M., Antoni, M., LeBlanc, W., y Schneiderman, N. (2003). Psychological distress is associated with decreased memory helper T-cell and B-cell counts pre-AIDS HIV seropositive men and women but only in those with low viral load. Psychosomatic Medicine, 65, 627-635.

Murphy, D., Johnston, K., y Martin, D. (2000). Barriers to Antiretroviral Adherence among HIV-Infected Adults. AIDS Patient Care and STDs, 14, 47-58.

Murphy, D., Wilson, C., Durako, S., Muenz, L., y Belzer, M. (2001). Antiretroviral medication adherence among the REACH HIVinfected adolescent cohort in the USA. AIDS Care, 13, 27-40.

O'Cleirigh, C., Ironson, G., Antoni, M., Pletcher, M.A., McGuffey, L., Balbin, E., Schneiderman, N., y Solomon, G. (2003). Emotional expression and depth processing of trauma and their relation to long-term survival in patients with HIV/AIDS. Journal of Psychosomatic Research, 54, 225-235.
O’Dowd, M., Biderman, D., y Mc Kegney, F. (1993). Incidence of suicidality in AIDS and HIV-positive patients attending a psychiatric outpatient program. Psychosomatics, 34, 33-40.

Orsulic-Jeras, S., Shepherd, J., y Britton, P. (2003). Counseling older adults with HIV/AIDS: A strength-based model of treatment. Journal of Mental Health Counseling, 25, 233-244.

Paterson, T., Shaw, W., y Semple, S. (2003). Reducing the sexual risk behaviors of HIV+ individuals: Outcome of a randomized controlled trial. Annals of Behavioral Medicine, 25, 137-145.

Penedo, F., González, J., Dahn, J., Antoni, M., Malow, R., Costa, P., y Schneiderman, N. (2003). Personality, quality of life and HAART adherence among men and women living with HIV/AIDS. Journal of Psychosomatic Research, 54, 271-278.

Pereira, D., Antoni, M., Danielson, A., Simon, T., Efantis-Potter, J., Carver, C., Duran, R., Ironson, G., Klimas, N., y O’Sullivan, M. (2003). Life stress and cervical squamous intraepithelial lesions in women with human papillomavirus and human immunodeficiency virus. Psychosomatic Medicine, 65, 427-434.

Perkins, D., Stern, R., Golden, R., Murphy, C., Naftolowitz, D., y Evans, D. (1994). Mood disorders in HIV infection: prevalence and risk factors in a nonepicenter of the AIDS epidemic. American Journal of Psychiatry, 151, 233-236.

Pomeroy, E., Green, D., y Van-Laningham, L. (2002). Couples who care: The effectiveness of a psychoeducational group intervention for HIV serodiscordant couples. Research on Social Work Practice, 12, 238252.

Power, R., Koopman, C., Volk, J., Israelski, D., Stone, L., Chesney, M., y Spiegel, D. (2003). Social support, substance use and denial in relationship to antiretroviral treatment adherence among HIV-infected persons. AIDS Patient Care and STD's, 17, 245-252.

Remien, R., Hirky, A., Johnson, M., Weinhardt, L., Whittier, D., y Minh-Le, G. (2003). Adherence to medication treatment: a qualitative study of facilitators and 
barriers among a diverse sample of HIV+ men and women in four U.S. cities. AIDS and Behavior, 7, 61-72.

Remien, R., Wagner, G., Dolezal, C., y Carballo, D. (2003). Levels and correlates of psychological distress in male couples of mixed HIV status. AIDS Care, 15, 525-538.

Remor, E. (2002). Valoración de la adhesión al tratamiento antirretroviral en pacientes VIH+. Psicothema, 14, 262-267.

Rietmeijer, C. (2003). The max for the minimum: Brief behavioral interventions can have important HIV prevention benefits. AIDS, 17, 1561-1562.

Robinson, F. (2002). Psycho-endocrine-immune response to mindfulness-based stress reduction in HIV-infected individuals. Dissertation Abstracts International, 63 (1-B), 179.

Roy, A. (2003). Characteristics of HIV patients who attempt suicide. Acta Psychiatrica Scandinavica, 107, 41-44.

Siegel, K., Karus, D., y Schrimshaw, E. (2000). Racial differences in attitudes toward protease inhibitors among older HIV-infected men. AIDS-Care, 12, 423-434.

Smith, M., Rapkin, B., Morrison, A., y Kammerman, S. (1997). Zidovudine adherence in persons with AIDS. The relation of patient beliefs about medication to self-termination of therapy. Journal of General Internal Medicine, 12, 216-223.

Smith, S., Rublein, J., Marcus, C., Brock, T., y Chesney, M. (2003). A medication selfmanagement program to improve adherence to HIV therapy regimens. Patient Education and Counseling, 50, 187-199.

Steele, R., y Grauer, D. (2003). Adherence to antiretroviral therapy for pediatric HIV infection: review of the literature and recom- mendations for research. Clinical Child and Family Psychology Review, 6, 17-30.

Sternhell, P., y Corr, M. (2002). Psychiatric morbidity and adherence to antiretroviral medication in patients with HIV/AIDS. Australian and New Zealand Journal of Psychiatry, 36, 528-533.

Stoskopf, C., Kim, Y., y Glover, S. (2001). Dual diagnosis: HIV and mental illness. A population-based study. Journal of Health Community Mental, 37, 469-479.

Turrina, C., Fiorazzo, A., Turano, A., Cacciani, P., Regina, C., Castelli, F., y Sachetti, E. (2001). Depressive disorders and personality variables in HIV positive and negative intravenous drug-users. Journal of Affective Disorders, 65, 45-53.

Ulla, S., y Remor, E. (2002). Psiconeuroimmunologia e infeccao por HIV: realidade ou ficcao? Psicologia: Reflexao e Critica, 15, 113-119.

Weinrich, J., Atkinson, J., McCutchan, J., y Grant, I. (1995). Is gender dysphoria dysphoric? Elevated depression and anxiety in gender dysphoric and nondysphoric homosexual and bisexual men in an HIV sample. Archives of Sexual Behavior, 24, 55-72.

Weiss, L., French, T., Finkelstein, R., Waters, M., Mukherjee, R., y Agins, B. (2003). HIVrelated knowledge and adherence to HAART. AIDS Care, 15, 673-679.

Witteveen, E., y van Ameijden, E. (2002). Drug users and HIV combination therapy (HAART): Factors which impede or facilitate adherence. Substance Use and Misuse, 37, 1905-1925.

Yarhouse, M. (2003). Working with families affected by HIV/AIDS. American Journal of Family Therapy, 31, 125-137. 